\title{
Monitoring insecticide resistance of adult and larval Aedes aegypti (Diptera: Culicidae) in Phnom Penh, Cambodia
}

Sebastien Boyer ${ }^{1,2^{*}} \mathbb{D}$, Pierre-Olivier Maquart ${ }^{1}$, Kalyan Chhuoy ${ }^{1}$, Kimhuor Suor $^{1}$, Moeun Chhum ${ }^{1}$, Kimly Heng ${ }^{1}$, Sokkeang Leng ${ }^{1}$, Didier Fontenille ${ }^{1,3}$ and Sebastien Marcombe ${ }^{4}$

\begin{abstract}
Background: Dengue fever is a major public health concern in Cambodia, with thousands of cases every year in urban, suburban and rural areas of the country. The main vector of dengue fever in Cambodia is Aedes aegypti. The organophosphate larvicide temephos and adulticides belonging to the pyrethroid family have been widely used for decades by public health authorities to fight dengue vectors, but resistance of Ae. aegypti to these insecticides has been previously described for Cambodia.
\end{abstract}

Methods: In order to adapt the vector control strategy presently used in Cambodia, we tested 14 adulticides belonging to the carbamate, organochlorine, organophosphate, and pyrethroid insecticide families and three larvicides [temephos, spinosad and Bacillus thuringiensis ser. israelensis (Bti)] belonging to three different insecticide families (organophosphates, spinosyns and entomopathogenic bacteria). The standard procedures used here to test the adults and larvae of an Ae. aegypti population from Phnom Penh followed World Health Organization guidelines.

Results: For adults, high mortality rates were observed with carbamate, organophosphate and organochlorine (with the exception of dichlorodiphenyltrichloroethane) insecticides (i.e. between 87.6 and 100\%), while low mortality rates were observed with all of the tested pyrethroid insecticides (i.e. between 1 and 35\%). For larvae, no resistance against Bti was detected [resistance ratio $\left.\left(R_{90}<1.6\right)\right]$, but moderate resistance was observed for temephos and spinosad $\left(R R_{90}<5.6\right)$.

Conclusions: The results of this study indicate that (i) Bti should be considered a serious alternative to temephos for the control of Ae. aegypti larvae; and (ii) the carbamate adulticides propoxur and bendiocarb should be employed instead of the widely used pyrethroid insecticides for the control of adult Ae. aegypti on land under mosaic farming and crop rotation in Cambodia, as the insects were found to be resistant to the latter types of insecticide. Research focusing on insecticide resistance and innovative and effective vector control strategies should be undertaken as a public health priority in Cambodia.

*Correspondence: sebastien.boyer@pasteur.fr

1 Medical and Veterinary Entomology Unit, Institut Pasteur du Cambodge, 5 Boulevard Monivong, Phnom Penh, Cambodia

Full list of author information is available at the end of the article

\section{Background}

Dengue fever is a major public health concern as it causes thousands of deaths every year in urban, suburban and rural tropical and sub-tropical areas [1]. Dengue virus, like Zika virus and chikungunya virus, is mainly transmitted by Aedes aegypti mosquitoes [2, 3]. In Cambodia, the Ministry of Health $(\mathrm{MoH})$ has been monitoring the original author(s) and the source, provide a link to the Creative Commons licence, and indicate if changes were made. The images or other third party material in this article are included in the article's Creative Commons licence, unless indicated otherwise in a credit line to the material. If material is not included in the article's Creative Commons licence and your intended use is not permitted by statutory regulation or exceeds the permitted use, you will need to obtain permission directly from the copyright holder. To view a copy of this licence, visit http://creativecommons.org/licenses/by/4.0/. The Creative Commons Public Domain Dedication waiver (http://creativeco mmons.org/publicdomain/zero/1.0/) applies to the data made available in this article, unless otherwise stated in a credit line to the data. 
incidence of dengue every week since the 1995 outbreak of this virus, which caused more than 400 deaths $[4,5]$. The most recent dengue epidemics in Cambodia were in 2007 (39,618 cases, 396 deaths), 2012 (42,362 cases, 189 deaths) and 2019 (68,597 cases, 48 deaths) (MoH, Phnom Penh, Cambodia). In 2018 and 2019, the capital of Cambodia, Phnom Penh, was affected by this disease as never before, with 9,445 and 9,298 cases, respectively [6]. The last arbovirus outbreak in Cambodia was the chikungunya epidemic in 2020, with a total of 1,722 cases throughout the 25 provinces of the country (Duong Veasna, personal communication).

Aedes aegypti is distributed throughout Phnom Penh [6] and in the Cambodian countryside [7-9], sometimes in sympatry with Aedes albopictus, another important arbovirus vector [6]. Hence, vector control targeting Ae. aegypti populations may have a wider public health impact. Temephos (Abate ${ }^{\circledR}$ ) has been the most broadly used insecticide in Cambodia since 1992 for vector control targeting larval stages of Ae. aegypti [10]. In Southeast Asia, adult Ae. aegypti are mainly targeted with pyrethroid insecticides, i.e. deltamethrin and permethrin, which have been used since the late 1980s in Cambodia [10-13]. In the late 1960s, Mouchet and Chastel [14] showed that Ae. aegypti was highly susceptible to dichlorodiphenyltrichloroethane (DDT), fenitrothion, malathion and diazinon insecticides, but resistant to dieldrin and gamma-hexachlorocyclohexane. Recently, Aedes resistance to temephos was investigated in several field studies [10, 15, 16]. In a study carried out in 2001 [15], an Aedes population from Phnom Penh was found to be resistant to the insecticide temephos tested at the World Health Organization (WHO) diagnostic dose, while a population from Kampong Cham province was susceptible. More recently, among seven Ae. aegypti populations from Phnom Penh and Kandal provinces, six were found to be resistant to temephos, with mortality rates ranging from 11 up to $89 \%[16,17]$. Finally, eight different urban and peri-urban Ae. aegypti populations were tested with temephos, deltamethrin and permethrin in 2016: all of the populations showed resistance to insecticides used for vector control in Cambodia [10].

In the present study, we tested 14 adulticides belonging to the carbamate, organochlorine, organophosphate and pyrethroid families, and three larvicides, belonging to the organophosphate, spinosyn, and entomopathogenic bacteria [Bacillus thuringiensis ser. Israelensis (Bti)] families, to determine one or more effective insecticides for vector control strategies adapted for use against Ae. aegypti in Phnom Penh.

\section{Methods}

\section{Mosquito sampling}

One population of Ae. aegypti (F1) was sampled in 2021 on the Institut Pasteur du Cambodge campus $\left(11^{\circ} 34^{\prime} 48.763 \mathrm{~N} ; 104^{\circ} 54^{\prime} 54.212 \mathrm{E}\right.$; World Geodetic System 84) in Phnom Penh. A susceptible United States Department of Agriculture (USDA) strain of Ae. aegypti was used as the control to test the effectiveness of the 17 insecticides [18].

Larvae and pupae were reared following standard conditions (i.e. temperature $27 \pm 1{ }^{\circ} \mathrm{C}$, relative humidity $75 \pm 25 \%$, photoperiod 12-h:12-h day/night) and were fed daily with half a teaspoon of fish food until adult emergence. Adult Aedes were reared under the same environmental conditions and fed with $10 \%$ sucrose solution. Female Ae. aegypti were blood-fed on laboratory-reared mice twice a week for $20 \mathrm{~min}$. F1 generation eggs were collected on white filter paper placed inside black cups half filled with water. Eggs were harvested daily and dried and stored in envelopes at a relative humidity of $50 \%$. F1 eggs were then immersed in water to obtain either larvae or adults for the different adult and larval bioassays.

\section{Adult A. aegypti bioassays}

Standard testing procedures for adult $A$. aegypti followed WHO guidelines [19]. Twenty-five females older than 3 days of age were used and tested in WHO test tubes.

Papers impregnated with specific concentrations of the insecticides were obtained from the Vector Control Research Unit at the University of Science, Penang, Malaysia. Papers impregnated with insecticides at the following concentrations were used for the bioassays [19]: the carbamates bendiocarb at $0.1 \%$ and propoxur at $0.1 \%$; the organochlorines DDT at $4 \%$ and dieldrin at 0.4 and $4 \%$; the organophosphates fenitrothion at $1 \%$, malathion at $0.8 \%$ and pirimiphos-methyl at $0.2 \%$; and the pyrethroids alpha-cypermethrin at $0.03 \%$, bifenthrin at $0.2 \%$, cyfluthrin at $0.15 \%$, deltamethrin at $0.03 \%$, etofenprox at $0.5 \%$, lambda-cyhalothrin at $0.03 \%$ and permethrin at $0.25 \%$. Two concentrations of dieldrin were used to distinguish between the susceptible, resistant heterozygous and resistant genotypes [19].

For both the Phnom Penh population and the USDA susceptible reference strain, four WHO tube tests, each with 25 adult females and one impregnated paper, were undertaken for each insecticide. Thus, a total of 100 mosquitoes were tested per strain for each insecticide, to which they were exposed for $1 \mathrm{~h}$. Mortality was assessed after $24 \mathrm{~h}$. 
As controls for each insecticide family, four kits were used with pyrethroid, organochlorine and organophosphate-carbamate control papers (WHO insecticide impregnated papers; Vector Control Research Unit), following the same test protocol described above for adult A. aegypti.

\section{Larval A. aegypti bioassays}

In accordance with WHO guidelines [19], late thirdinstar larvae of the F1 generation were used to determine the susceptibility of the mosquito larvae to the three larvicides: temephos (PESTANAL, analytical grade, $250 \mathrm{mg}$; Sigma-Aldrich, St Louis, MO); Bti (VectoBac strain AM65-52; Valent BioSciences, Thailand); and spinosad (Sigma-Aldrich). Larvae showing any abnormalities were removed from the experiment. To determine the lethal dose $(\mathrm{LD})$ required to kill $50 \%\left(\mathrm{LD}_{50}\right)$ and $90 \%\left(\mathrm{LD}_{90}\right)$ of the larvae, seven different concentrations were tested for each larvicide [20]. For the bioassays, temephos was used at $0,5,10,20,30,50,100$ and $200 \mu \mathrm{g} / \mathrm{L}$, spinosad at 0,1 , $5,10,25,50,100$ and $200 \mu \mathrm{g} / \mathrm{L}$ and Bti at 0, 50, 100, 200, 400, 600, 800 and $1000 \mu \mathrm{g} / \mathrm{L}$.

The positive control comprised a USDA susceptible reference strain with insecticide [18], and the negative control larvae in water without insecticide. For each of the tested insecticide concentrations and controls, four replicates (four 400-mL plastic goblets containing $200 \mathrm{~mL}$ of insecticide solution) with 25 larvae per replicate were used.

\section{Data management and statistical analysis}

For adults, mortality was determined $24 \mathrm{~h}$ post-exposure. For the larvae, $\mathrm{LD}_{50}$ and $\mathrm{LD}_{90}$ were obtained by plotting mortality using log probit analysis with $\mathrm{R}$ Core Team software [21]. The $\mathrm{LD}_{50}$ and $\mathrm{LD}_{95}$ results obtained for the field strains were then divided by the results obtained using the USDA strain to calculate the resistance ratio (RR) for each field population.

\section{Results}

\section{Adult bioassays}

All of the negative controls realized with the control impregnated papers (pyrethroid, organochlorine and organophosphate-carbamate control papers) showed $0 \%$ mortality after $24 \mathrm{~h}$. For the positive controls, $100 \%$ mortality of the USDA strain was observed for all of the insecticides. For the field strain, high mortality rates were observed with the carbamate, organochlorine (except for DDT) and organophosphate insecticides, while low mortality rates were observed with all of the pyrethroid insecticides.

For the carbamates, adult Ae. aegypti mortalities were $96.1 \pm 3.3 \%$ for $0.1 \%$ bendiocarb and $97.8 \pm 2.6 \%$ for $0.1 \%$ propoxur. For the organochlorines, the mortality rates were $0 \%$ for $4 \%$ DDT and $87.6 \pm 2.1 \%$ and $97.8 \pm 4.3 \%$, respectively, for $0.4 \%$ and $4 \%$ dieldrin. For the organophosphate insecticides, the adult mortalities were $91.1 \pm 5.9 \%$ for $0.8 \%$ malathion, $99.1 \pm 1.9 \%$ for $1 \%$ fenitrothion and $100 \%$ for $0.2 \%$ pirimiphos-methyl. For the pyrethroid insecticides, the adult mortalities

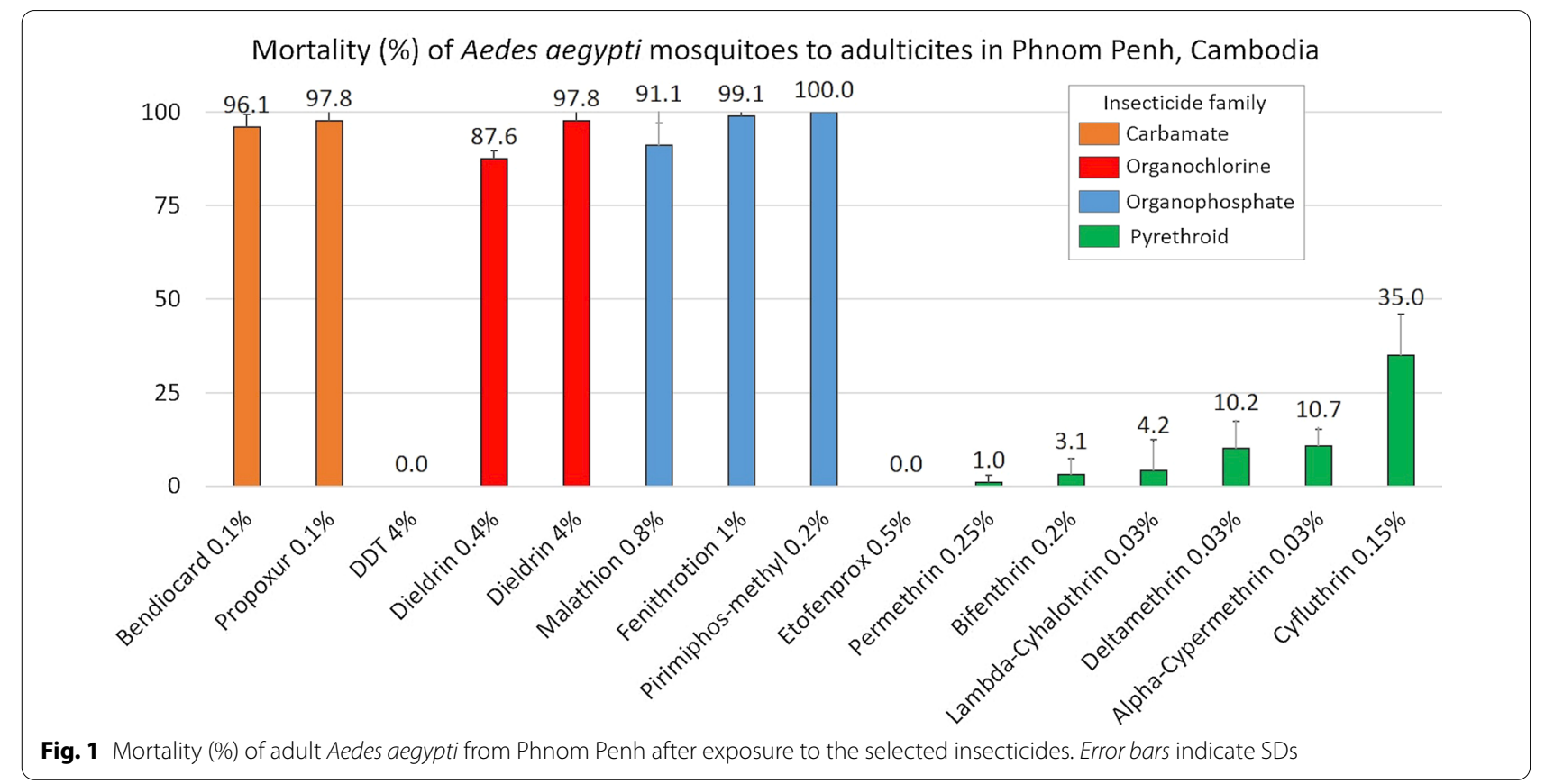



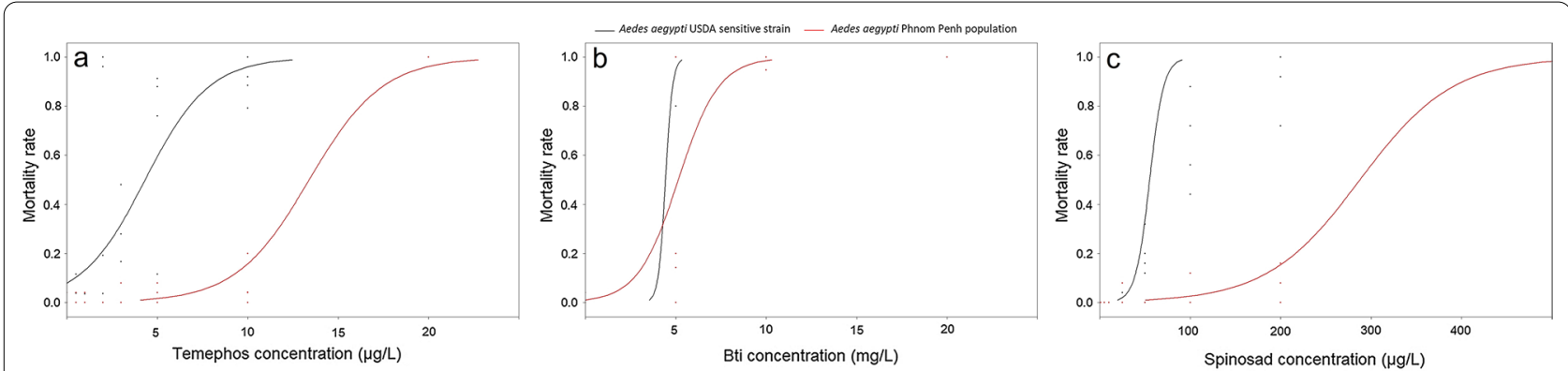

Fig. 2 Mortality rate of Aedes aegypti larvae from Phnom Penh exposed to a spinosad ( $\mu \mathrm{g} / \mathrm{L})$, $\mathbf{b}$ temephos $(\mu \mathrm{g} / \mathrm{L})$, and $\mathbf{c}$ Bacillus thuringiensis ser. Israelensis (Bti) (mg/L). Black lines Susceptible reference strain, red lines field population

were $0 \%$ for $0.5 \%$ etofenprox, $1 \pm 1.9 \%$ for $0.25 \%$ permethrin, $3.1 \pm 4.1 \%$ for $0.2 \%$ bifenthrin, $4.2 \pm 8.3 \%$ for $0.03 \%$ lambda-cyhalothrin, $10.2 \pm 7.1 \%$ for $0.03 \%$ deltamethrin, $11 \pm 4.5 \%$ for $0.03 \%$ alpha-cypermethrin and $35 \pm 11 \%$ for $0.15 \%$ cyfluthrin (Fig. 1).

\section{Larval bioassays}

The $\mathrm{LD}_{50}$ for larvae of the USDA strain was $4.3 \pm 0.2 \mu \mathrm{g} / \mathrm{L}$ for temephos, $55.2 \pm 2.4 \mu \mathrm{g} / \mathrm{L}$ for spinosad and $4.4 \pm 0.1 \mathrm{mg} / \mathrm{L}$ for Bti. The $\mathrm{LD}_{90}$ was $8.2 \pm 0.5 \mu \mathrm{g} / \mathrm{L}$ for temephos, $72.3 \pm 4.5 \mu \mathrm{g} / \mathrm{L}$ for spinosad and $4.9 \pm 0.02 \mathrm{mg} / \mathrm{L}$ for Bti.

For larvae of the Phnom Penh population of Ae. aegypti, the $\mathrm{LD}_{50}$ and $\mathrm{LD}_{90}$ for temephos were $13.6 \pm 0.7 \mu \mathrm{g} / \mathrm{L}$ and $17.9 \pm 0.8 \mu \mathrm{g} / \mathrm{L}$, respectively, representing an $R R_{50}$ of 3.1 and an $\mathrm{RR}_{90}$ of 2.2 (Table 1). For spinosad, the $\mathrm{LD}_{50}$ and $\mathrm{LD}_{90}$ were $287.8 \pm 29.7 \mu \mathrm{g} / \mathrm{L}$ and $401.2 \pm 49.9 \mu \mathrm{g} / \mathrm{L}$, respectively, representing the highest $R$ Rs, i.e. an $R_{50}$ of 5.2 and an $R R_{90}$ of 5.6. The lowest $R R$ s were obtained with Bti $\left(\mathrm{RR}_{50}\right.$ of 1.2 and $\mathrm{RR}_{90}$ of $1.6 ; \mathrm{LD}_{50}$ of $5.2 \pm 0.4 \mathrm{mg} / \mathrm{L}$ and $\mathrm{LD}_{90}$ of $7.6 \pm 0.6 \mathrm{mg} / \mathrm{L}$ ) (Fig. 2).

\section{Discussion}

In Cambodia, the main insecticides used to control mosquitoes are temephos, which is used against larvae, and permethrin and deltamethrin, which are used against adults. This study, like many previous ones [10, 14-16], demonstrated the resistance of Ae. aegypti to these insecticides. As the vector control of widely distributed species of mosquitoes in Cambodia is mainly based on the use of the larvicide temephos ( $235 \mathrm{t}$ were imported into the country in 2020, according to a $\mathrm{MoH}$ report), we decided to assess the susceptibility of larvae of the Phnom Penh population tested here to two other larvicides that are also used worldwide, spinosad and Bti.

For spinosad, we observed a moderate resistance. Resistance to spinosad was also observed in an Ae. aegypti population in Lao People's Democratic Republic (PDR) that was also resistant to temephos [20]. Furthermore, in Brazil, similar results were also obtained for spinosad, with mortality of larvae exceeding $80 \%$ and an $\mathrm{RR}_{50}$ ranging from 2.5 to 4.1 for populations that were highly resistant to temephos (for temephos, the $R_{50}$ ranged from 6.5 to 89.8 for all but one of the tested populations) [22]. The insecticide spinosad disrupts nicotinic acetylcholine receptors, which leads to paralysis, followed by death. The mechanism of resistance that has thus far been described is a mutation of the beta subunit of a gene coding for a nicotinic acetylcholine receptor [23]. It would be interesting to determine if the Phnom Penh population has a mutation within the same area of its genome, or to know if this phenotypic resistance is due to another selected mechanism (i.e. cross-resistance) with temephos (i.e. enzymatic detoxification).

The Ae. aegypti population was susceptible to Bti. This result agrees with those previously reported for $A e$.

Table 1 Mean larval Lethal Dose $\left(\mathrm{LD}_{50}\right.$ and $\left.\mathrm{LD}_{90}\right) \pm \mathrm{SE}$ of Phnom Penh population of Aedes aegypti with temephos, spinosad and Bti

\begin{tabular}{|c|c|c|c|c|c|c|}
\hline \multirow[t]{2}{*}{ Insecticides } & \multicolumn{2}{|l|}{ USDA } & \multicolumn{4}{|l|}{ Phnom Penh } \\
\hline & $\mathrm{LD}_{50}$ & $\mathrm{LD}_{90}$ & $\mathrm{LD}_{50}$ & $\mathrm{RR}_{50}$ & $\mathrm{LD}_{90}$ & $\mathrm{RR}_{90}$ \\
\hline Temephos ( $\mu \mathrm{g} / \mathrm{L})$ & $4.3 \pm 0.2(3.9-4.7), n=809$ & $8.2 \pm 0.5(7.2-9.2), n=809$ & $13.6 \pm 0.7(12.2-15.0), n=798$ & 3.1 & $17.9 \pm 0.8(16.3-19.5), n=798$ & 82.2 \\
\hline Spinosad ( $\mu \mathrm{g} / \mathrm{L})$ & $55.2 \pm 2.4(50.4-59.9), n=746$ & $72.3 \pm 4.5(63.4-81.1), n=746$ & $\begin{array}{l}287.8 \pm 29.7(277.6-298.0) \\
n=750\end{array}$ & 5.2 & $\begin{array}{l}401.2 \pm 49.9(303.3-499.1) \\
n=750\end{array}$ & 5.6 \\
\hline Bti (mg/L) & $4.4 \pm 0.1(4.2-4.6), n=807$ & $4.9 \pm 0.02(4.8-5.0), n=807$ & $5.2 \pm 0.42 .8-7.6), n=709$ & 1.2 & $7.6 \pm 0.6(6.4-8.8), n=709$ & 1.6 \\
\hline
\end{tabular}


aegypti in Cambodia, when Bti was successfully tested around Phnom Penh in 2005 and 2016 [16, 17]. Furthermore, in Lao PDR, promising results were found for Bti against wild temephos-resistant populations of $A e$. aegypti in the laboratory and in semi-field trials [20]. In light of these results, Lao PDR MoH changed its policy and decided to use Bti instead of temephos in 2019. Bti appears to be a good alternative to temephos for use as a larvicide in Cambodia.

One of the most important findings of our study is the very low mortality, and therefore high resistance, of the Ae. aegypti adults to all the tested pyrethroids, including the two routinely used in Cambodia, permethrin and deltamethrin. Resistance to these two pyrethroids has been previously described, and their ineffectiveness recognized [10, 24]. The two most widely recognized mechanisms of insecticide resistance are voltage-gated channel modification (knockdown resistance mutation; $k d r)$ and the overproduction of detoxification enzymes $[25,26]$. Even though pyrethroids target sodium channels [27], resistance mechanisms are not specific to this particular insecticide family but to the structural conformation of each pyrethroid insecticide. This might explain why the same phenotypic pattern of resistance is rarely observed between type I and II pyrethroid forms and pseudo-pyrethroids that are non-ester pyrethroids, e.g. etofenprox [28]. Observed resistance to all pyrethroids as well as to the organochlorine DDT suggests that several $k d r$ mutations have been selected as a result of mosquitoes coming into repeated contact over decades with different insecticides that synergize when combined [29,30]. Indeed, very high levels of pyrethroid resistance have been reported, with some individuals carrying two (S989P + V1016G) or three mutations (S989P + V1016G + F1534C) in their sodium channel genes [31, 32]. The description of several $k d r-$ associated mutations seems to validate this hypothesis $[27,31,33]$. Studies have shown the presence of different $k d r$ mutations in Southeast Asia [29, 34, 35]. Hence, the use of pyrethroids in Cambodia, Lao PDR [20] and Thailand $[32,36]$ is clearly compromised.

Furthermore, although organochlorine and organophosphate insecticides, with the exception of DDT, cause high mortality in adult Ae. aegypti, they cannot be recommended for vector control due to their broad spectrum of action and persistence in the environment. Fortunately, the two tested carbamates, bendiocarb and propoxur, were effective at killing the mosquitoes. In an Australian study [37] carried out in 1999, Ae. aegypti was found to be resistant to bendiocarb, while there was no evidence that it was resistant to pyrethroids. A decrease in susceptibility of this species to bendiocarb was also observed in Colombia [38], Malaysia [39],
Trinidad and Tobago [40], and Mexico [41]. However, no resistance to bendiocarb was detected in this species in Costa Rica [42] or in a study carried out in Mexico [43]. Ae. aegypti showed no resistance to propoxur in studies carried out in Australia [37], Colombia [44], Mexico [43] and Panama [45]. Resistance to propoxur was found in three of the ten tested Ae. aegypti populations in a study undertaken in Colombia [38], and was more frequent in Ae. aegypti in Malaysia [39]. In sum, bendiocarb and propoxur are considered good potential alternative insecticides for the control of Ae. aegypti during outbreak events in Cambodia.

\section{Conclusions}

At least two conclusions have emerged from this work: further study of insecticide resistance, a major concern in Cambodia, should be a public health priority for the country; alternative and innovative vector control strategies should be developed for Cambodia. The study of insecticide resistance, including a focus on molecular bases and biomarkers, which is presently not an axis of research in Cambodia, should be implemented as soon as possible to help orientate the $\mathrm{MoH}$ in its fight against vector species of mosquito. Recently developed effective vector control strategies which use Wolbachia against Ae. aegypti could be a promising tool for use in Cambodia and throughout Southeast Asia. Finally, Bti should be considered a very good candidate alternative insecticide to temephos for the control of Ae. aegypti larvae, and the adulticides propoxur and bendiocarb (carbamate) for the control of Ae. aegypti on land under mosaic farming or crop rotation.

\section{Authors' contributions}

Conception and design: SB and DF. Acquisition and analysis: KC, KS, MC, KH and SL. Interpretation of the data and writing of the manuscript: SB, POM, DF and SM. All the authors read and approved the final manuscript.

\section{Funding}

The research was funded by the Institut Pasteur du Cambodge; ZIKAlliance, which is funded by the H2020 program of the European Union (award no. 734548); the FSPI-SUPREV project (FSPI 2019-17); and a Calmette and Yersin post-doctoral grant.

\section{Availability of data and materials}

All data generated or analyzed during this study are included in this published article and its additional files.

\section{Declarations}

Ethics approval and consent to participate Not applicable.

Consent for publication

Not applicable.

Competing interests

The authors declare that they have no competing interests. 


\section{Author details}

${ }^{1}$ Medical and Veterinary Entomology Unit, Institut Pasteur du Cambodge, 5 Boulevard Monivong, Phnom Penh, Cambodia. ${ }^{2}$ International Pasteur Institute Network, Institut Pasteur, Paris, France. ${ }^{3}$ MIVEGEC, Université de Montpellier, CNRS, IRD (Institut de Recherche Pour Le Développement), 911 Avenue Agropolis, 34394 Montpellier, France. ${ }^{4}$ Medical Entomology Unit, Ministry of Health, Institut Pasteur du Laos, Vientiane, Lao People's Democratic Republic.

Received: 14 October 2021 Accepted: 8 January 2022

Published online: 31 January 2022

\section{References}

1. Guzman A, Istúriz RE. Update on the global spread of dengue. Int J Antimicrobial Agents. 2010;36:S40-2.

2. Souza-Neto JA, Powell JR, Bonizzoni M. Aedes aegypti vector competence studies: a review. Infect Genet Evol. 2019;67:191-209.

3. Pereira-dos-Santos T, et al. A systematic review: is Aedes albopictus an efficient bridge vector for zoonotic arboviruses? Pathogens. 2020;9(4):266.

4. Huy R, et al. National dengue surveillance in Cambodia 1980-2008: epidemiological and virological trends and the impact of vector control. Bull World Health Organ. 2010;88:650-7.

5. Vong $S$, et al. Dengue incidence in urban and rural Cambodia: results from population-based active fever surveillance, 2006-2008. PLoS Negl Trop Dis. 2010;4(11):e903.

6. Maquart PO, Fontenille D, Boyer S. Recent and massive invasion of Aedes (Stegomyia) albopictus (Skuse, 1894) in Phnom Penh Cambodia. Parasit Vectors. 2021;14(1):1-3.

7. Boyer S, et al. High diversity of mosquito vectors in Cambodian primary schools and consequences for arbovirus transmission. PLOS ONE. 2020;15(6):e0233669.

8. Boyer $\mathrm{S}$, et al. Host-feeding preference and diel activity of mosquito vectors of the Japanese encephalitis virus in rural Cambodia. Pathogens. 2021;10(3):376.

9. Maquart PO, et al. Checklist of the mosquito fauna (Diptera: Culicidae) of Cambodia. Parasites. 2021;28 (in press).

10. Boyer S, et al. Resistance of Aedes aegypti (Diptera: Culicidae) populations to deltamethrin, permethrin, and temephos in Cambodia. Asia Pac J Public Health. 2018;30(2):158-66.

11. Marcombe $S$, et al. Distribution of insecticide resistance and mechanisms involved in the arbovirus vector Aedes aegypti in Laos and implication for vector control. PLoS Negl Trop Dis. 2019;13(12):e0007852.

12. Kawada $\mathrm{H}$, et al. Insecticidal and repellent activities of pyrethroids to the three major pyrethroid-resistant malaria vectors in western Kenya. Parasit Vectors. 2014;7(1):1-9.

13. Jirakanjanakit $\mathrm{N}$, et al. Insecticide susceptible/resistance status in Aedes (Stegomyia) aegypti and Aedes (Stegomyia) albopictus (Diptera: Culicidae) in Thailand during 2003-2005. J Econ Entomol. 2007;100:545-50.

14. Mouchet J, Chastel C. Resistance to insecticides in Aedes aegypti $\mathrm{L}$. and Aedes albopictus in Phnom-Penh (Cambodia). Med Trop. 1966;26(5):505-15.

15. Polson KA, et al. Susceptibility of two Cambodian population of Aedes aegypti mosquito larvae to temephos during 2001. Dengue Bull. 2001;25:79-83.

16. Setha T, Chantha N, Socheat D. Efficacy of Bacillus thuringiensis israelensis, VectoBac ${ }^{\circledR}$ WG and DT, formulations against dengue mosquito vectors in cement potable water jars in Cambodia. Southeast Asian J Trop Med Public Health. 2007;38:261-8.

17. Setha T, et al. Bacterial larvicide, Bacillus thuringiensis israelensis strain AM 65-52 water dispersible granule formulation impacts both dengue vector, Aedes aegypti (L.) population density and disease transmission in Cambodia. PLoS Negl Trop Dis. 2016;10:e0004973.

18. Kuno G. Early history of laboratory breeding of Aedes aegypti (Diptera: Culicidae) focusing on the origins and use of selected strains. J Med Entomol. 2010;47:957-71.

19. World Health Organization (WHO). Monitoring and managing insecticide resistance in Aedes mosquito populations. Interim guidance for entomologists. World Health Organization; 2016.
20. Marcombe S, et al. Alternative insecticides for larval control of the dengue vector Aedes aegypti in Lao PDR: insecticide resistance and semi-field trial study. Parasit Vectors. 2018;11:616.

21. R Core Team (2020). R: a language and environment for statistical computing. R Foundation for Statistical Computing, Vienna, Austria.

22. dos Santos Dias L, et al. Toxicity of spinosad to temephos-resistant Aedes aegypti populations in Brazil. PLoS ONE. 2017;12(3):e0173689.

23. Lan J, et al. Identification of the Aedes aegypti $\mathrm{nAChR}$ gene family and molecular target of spinosad. Pest Manag Sci. 2021;77(4):1633-41.

24. Marcombe $\mathrm{S}$, et al. Insecticide resistance in the dengue vector Aedes aegypti from Martinique: distribution, mechanisms and relations with environmental factors. PLoS ONE. 2012;7(2):e30989.

25. Dusfour l, et al. Multiple insecticide resistance in Aedes aegypti (Diptera: Culicidae) populations compromises the effectiveness of dengue vector control in French Guiana. Mem Inst Oswaldo Cruz. 2011;106(3):346-52.

26. Moyes, et al. Contemporary status of insecticide resistance in the major Aedes vectors of arboviruses infecting humans. PLoS Negl Trop Dis. 2017;11(7):e0005625.

27. Li CX, et al. Relationship between insecticide resistance and kdr mutations in the dengue vector Aedes aegypti in ssouthern China. Parasit Vectors. 2015;8(1):1-9.

28. Schleier III JJ, Peterson RKD. Pyrethrins and pyrethroid insecticides. In Oscar Lopez, Jose G. Fernfmdez-Bolafios (Eds.), Green trends in insect control. Royal Society of Chemistry; 2011.

29. Kawada $\mathrm{H}$, et al. Widespread distribution of a newly found point mutation in voltage-gated sodium channel in pyrethroid-resistant Aedes aegypti populations in Vietnam. PLoS Negl Trop Dis. 2009;3(10):e527.

30. Brengues $C$, et al. Pyrethroid and DDT cross-resistance in Aedes aegypti is correlated with novel mutations in the voltage-gated sodium channel gene. Med Vet Entomol. 2003;17(1):87-94.

31. Hirata $\mathrm{K}$, et al. A single crossing-over event in voltage-sensitive $\mathrm{Na}+$ channel genes may cause critical failure of dengue mosquito control by insecticides. PLoS Negl Trop Dis. 2014;8(8):e3085.

32. Plernsub S, et al. Additive effect of knockdown resistance mutations, S989P, V1016G and F1534C, in a heterozygous genotype conferring pyrethroid resistance in Aedes aegypti in Thailand. Parasit Vectors. 2016;9(1):1-7.

33. Fernando SD, et al. First report of V1016G and S989P knockdown resistant ( $k d r)$ mutations in pyrethroid-resistant Sri Lankan Aedes aegypti mosquitoes. Parasit Vectors. 2018;11:526.

34. Stenhouse SA, et al. Detection of the V1016G mutation in the voltagegated sodium channel gene of Aedes aegypti (Diptera: Culicidae) by allele-specific PCR assay, and its distribution and effect on deltamethrin resistance in Thailand. Parasit Vectors. 2013;6:253.

35. Yanola J, et al. High-throughput assays for detection of the F1534C mutation in the voltage-gated sodium channel gene in permethrin-resistant Aedes aegypti and the distribution of this mutation throughout Thailand. Trop Med Int Health. 2011;16(4):501-9.

36. Chuaycharoensuk, et al. Frequency of pyrethroid resistance in Aedes aegypti and Aedes albopictus (Diptera: Culicidae) in Thailand. J Vector Ecol. 2011;36(1):204-12.

37. Canyon DV, Hii JLK. Insecticide susceptibility status of Aedes aegypti (Diptera: Culicidae) from Townsville. Australian J Entomol. 1999;38(1):40-3.

38. Ocampo CB, et al. Insecticide resistance status of Aedes aegypti in 10 localities in Colombia. Acta Trop. 2011;118(1):37-44.

39. Loke SR, et al. Insecticide susceptibility status of field-collected Aedes (Stegomyia) aegypti (L.) from a dengue endemic site in Shah Alam, Selangor, Malaysia. Southeast Asian J Trop Med Public Health. 2012;43(1):34.

40. Polson KA, et al. Characterisation of DDT and pyrethroid resistance in Trinidad and Tobago populations of Aedes aegypti. Bull Entomol Res. 2011;101(4):435.

41. Deming $R$, et al. Spatial variation of insecticide resistance in the dengue vector Aedes aegypti presents unique vector control challenges. Parasit Vectors. 2016;9(1):1-10.

42. Bisset JA, et al. Insecticide resistance in two Aedes aegypti (Diptera: Culicidae) strains from Costa Rica. J Med Entomol. 2013;50(2):352-61.

43. Kuri-Morales PA, et al. Insecticide susceptibility status in Mexican populations of Stegomyia aegypti (= Aedes aegypti): a nationwide assessment. Med Vet Entomol. 2018;32(2):162-74.

44. Santacoloma $L$, et al. Estado de la susceptibilidad de poblaciones naturales del vector del dengue a insecticidas en trece localidades de Colombia. Biomedica. 2012;32(3):333-43. 
45. Cáceres $L$, et al. Determinación de la sensibilidad a insecticidas organofosforados, carbamato y piretroides en poblaciones de Aedes aegypti Linneaus, 1762 (Díptera: Culicidae) de Panamá. Biomedica. 2013;33(1):70-81.

\section{Publisher's Note}

Springer Nature remains neutral with regard to jurisdictional claims in published maps and institutional affiliations. 